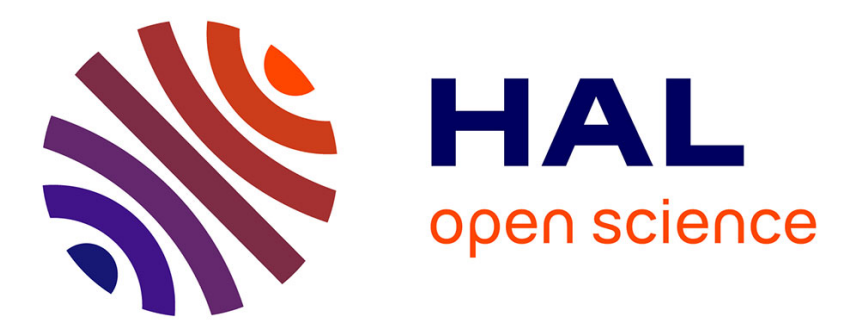

\title{
Enhancement mode HEMT using fluorine implantation below the channel
}

Saleem Hamady, Frédéric Morancho, Bilal Beydoun, Patrick Austin, Mathieu

Gavelle

\section{- To cite this version:}

Saleem Hamady, Frédéric Morancho, Bilal Beydoun, Patrick Austin, Mathieu Gavelle. Enhancement mode HEMT using fluorine implantation below the channel. European Physical Journal: Applied Physics, 2015, 70 (3), pp.30102. 10.1051/epjap/2015150062 . hal-01955606

\section{HAL Id: hal-01955606 https://hal.laas.fr/hal-01955606}

Submitted on 14 Dec 2018

HAL is a multi-disciplinary open access archive for the deposit and dissemination of scientific research documents, whether they are published or not. The documents may come from teaching and research institutions in France or abroad, or from public or private research centers.
L'archive ouverte pluridisciplinaire HAL, est destinée au dépôt et à la diffusion de documents scientifiques de niveau recherche, publiés ou non, émanant des établissements d'enseignement et de recherche français ou étrangers, des laboratoires publics ou privés. 


\title{
Enhancement mode HEMT using fluorine implantation below the channel ${ }^{\star}$
}

\author{
Saleem Hamady ${ }^{1,2,3, a}$, Frédéric Morancho ${ }^{1,4}$, Bilal Beydoun ${ }^{3}$, Patrick Austin ${ }^{1,4}$, and Mathieu Gavelle ${ }^{5}$ \\ ${ }^{1}$ CNRS, LAAS, 7 avenue du Colonel Roche, 31400 Toulouse, France \\ ${ }^{2}$ Université de Toulouse, LAAS, 31400 Toulouse, France \\ ${ }^{3}$ GET/LPE, Lebanese University, Hadath Campus, Beirut, Lebanon \\ ${ }^{4}$ Université de Toulouse, UPS, LAAS, 31400 Toulouse, France \\ ${ }^{5}$ CEA Tech Midi-Pyrénées, 135 avenue de Rangueil, INSA Bât. 8, 31400 Toulouse, France
}

Received: 31 January 2015 / Revised in final form: 28 April 2015 / Accepted: 15 May 2015 Published online: 24 June 2015 - (C) EDP Sciences 2015

\begin{abstract}
Gallium nitride based high electron mobility transistors (HEMT) are powerful candidates for high frequency and high power applications. While switching applications demand normally-off operation, these devices are normally-on. Recent normally-off HEMTs were demonstrated by implanting fluorine above the channel, in the barrier layer. During implantation, fluorine ions penetrate into the channel and cause mobility degradation. In this paper, we propose and simulate an alternative approach in which fluorine ions are implanted below the channel of the HEMT rather than above it. The simulation tool ATLAS is calibrated using experimental data from a real HEMT device. Simulation results have shown that implanting fluorine ions below the channel is capable of achieving normally-off operation. When compared to the implantation in the barrier layer, the proposed approach offers better confinement for the two dimensional electron gas (2DEG) below the gate, eliminates the scattering of fluorine ions with channel electrons and is more efficient when it comes to the fluorine concentration required to achieve a desired threshold voltage. This technique neither affects the breakdown voltage nor the off-state current.
\end{abstract}

\section{Introduction}

AlGaN/GaN HEMTs are very promising candidates for high frequency applications with high power and low noise, such as microwave and millimeter wave communications, imaging and radars [1]. With the high field strength offered by GaN and the high mobility of the two dimensional electron gas (2DEG) in the HEMT, this device can reach high breakdown voltage with low ON-state resistance and high switching frequency, surpassing the limitation of conventional silicon devices. In the conventional HEMT, the triangular well, formed at $\mathrm{AlGaN} / \mathrm{GaN}$ interface, is below the Fermi level at equilibrium. This makes the channel populated with electrons at zero gate voltage, hence making the HEMT normally-on. However, for power switching applications, normally-off operation is strongly required [2]. In order to achieve normally-off operation, the triangular well must be lifted above the Fermi level. Several structures have been proposed for the realization of normally-off AlGaN/GaN HEMTs, such as the recessed gate structures [3], fluorine ion treatment [4], PN junction

\footnotetext{
${ }^{a}$ e-mail: shamady@laas.fr

* Contribution to the topical issue "Electrical Engineering Symposium (SGE 2014) - Elected submissions", edited by Adel Razek
}

gate structure [5], thin AlGaN barrier [6], AlN/GaN structures [7] and HEMT with InGaN cap layer [8].

In the normally-off HEMT reported in reference [4], fluorine ions are implanted above the channel, in the barrier layer (AlGaN layer). According to this approach, during implantation, small amount of fluorine ions penetrate into the channel formed at the $\mathrm{AlGaN} / \mathrm{GaN}$ interface, presenting themselves as impurities that could lead to mobility degradation [9]. In this paper, we propose the implantation of fluorine ions below the channel (in the GaN layer) under the AlGaN/GaN interface, only below the gate electrode. To obtain this structure, the following process can be used: after growing the buffer and GaN layers, fluorine ions are implanted. Then the channel layer of $\mathrm{GaN}(5-50 \mathrm{~nm})$ is grown and above all comes the AlGaN layer (barrier layer). With fluorine being implanted before the growth of the channel layer, and with the thickness of the channel layer being greater than the thickness of the 2DEG ( $\sim 5 \mathrm{~nm})$, fluorine ions will be buried away from the channel electrons and hence, no scattering will occur.

\section{Simulation strategy}

ATLAS, a physically based TCAD simulation tool from Silvaco, is used to simulate the electrical characteristics 


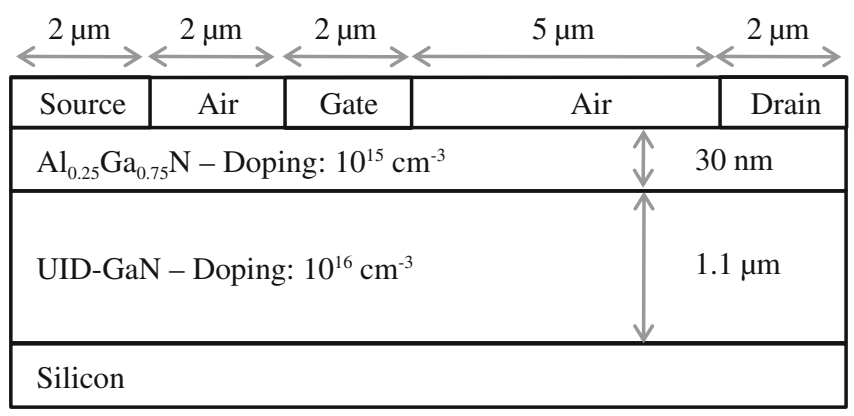

Fig. 1. Geometrical and technological parameters used for the normally-on HEMT structure and the calibration of the TCAD simulations.

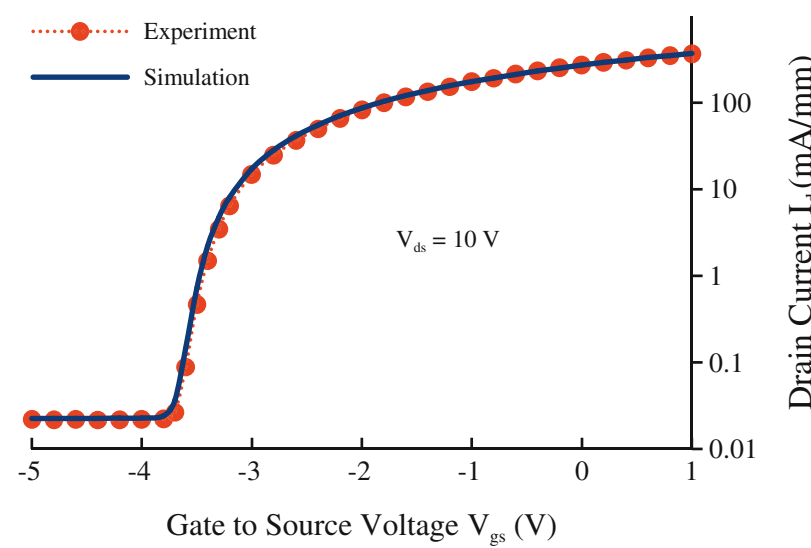

Fig. 2. Comparison of experimental and simulated $I_{\mathrm{d}}\left(V_{\mathrm{gs}}\right)$ transfer characteristics.

of the proposed HEMT structure. Physical models of the simulator are based on Shockley-Read Hall recombination, Fermi-Dirac statistics and field-dependent mobility [10]. The simulator is calibrated by using physical and geometrical parameters of a real normally-on HEMT device. The cross-section of this device is shown in Figure 1. The structure of the epi-wafer comprises a $30 \mathrm{~nm}$ unintentionally doped (UID) AlGaN barrier layer followed by $1.1 \mu \mathrm{m}$ UID-GaN layer, grown on a $\mathrm{Si}(111)$ substrate. The $\mathrm{Al}$ content in the barrier layer is $25 \%$. The distances between the source and the gate, the gate and the drain, and the gate width are $2 \mu \mathrm{m}, 2 \mu \mathrm{m}$ and $5 \mu \mathrm{m}$ respectively. The simulated transfer characteristics $I_{\mathrm{d}}\left(V_{\mathrm{gs}}\right)$, compared with the experimental ones, are presented in Figure 2. A good match is observed for the threshold voltage $\left(V_{\mathrm{th}}\right)$ and the transconductance $\left(g_{\mathrm{m}}\right)$.

During calibration, the energy and the concentration of the acceptor traps as well as the density of the 2DEG were tuned. Some of the parameters used for the simulation are illustrated in Table 1.

In Section 3, fluorine ions are added below or above the channel of the calibrated structure (Fig. 1). To simulate the effect of the implanted fluorine ions, negative charge is added inside the GaN (or AlGaN) layer. To perform that in ATLAS, the GaN (or AlGaN) layer is split into two layers with the same material. This approach generates an interface (GaN/GaN or AlGaN/AlGaN homo-interface)
Table 1. HEMT simulation parameters.

\begin{tabular}{cc}
\hline Density of acceptor traps & $10^{17} \mathrm{~cm}^{-3}$ \\
in GaN and AlGaN layers & \\
Energy of acceptor traps & $0.41 \mathrm{eV}$ \\
as measured from the & \\
conduction band & $K\left(P_{\mathrm{GaN}}-P_{\mathrm{AlGaN}}\right) / q \mathrm{~cm}^{-2}$ \\
Bound charge density & 0.61 \\
Fitting parameter $K$ & \\
\hline
\end{tabular}

\begin{tabular}{|c|c|c|c|}
\hline Source & Gate I & & Drain \\
\hline AlGaN & \multicolumn{3}{|c|}{$\longrightarrow$ Cut } \\
\hline $\mathrm{GaN}$ & $i$ & \multicolumn{2}{|c|}{$\uparrow \mathrm{d}$} \\
\hline $\mathrm{GaN}$ & Fluorine Ions & $\begin{array}{c}\mathrm{GaN} / \mathrm{GaN} \\
\text { interface }\end{array}$ & \\
\hline Silicon & & & \\
\hline
\end{tabular}

Fig. 3. Strategy used to simulate the effect of fluorine ions using ATLAS.

inside the layer. At this interface, fixed negative charge is added as shown in Figure 3. It should be mentioned that the charge is added only below the gate electrode and not all over the homo-interface.

The position of the charge can be varied, by changing the thickness of the split layer. The profile of the concentration of fluorine ions, resulting from this approach, differs from the experimental profile. However, the effect on the local potential can be imitated by varying the concentration of the negative charge at the interface. The variation in the local potential causes the shift in threshold voltage. Therefore, this model can predict the shift in the threshold resulting from fluorine implantation. The use of a fluorine equivalent interface charge was used by [11] to derive a comprehensive analytical model for the threshold voltage of fluorinated MOS-HEMTs.

To confirm the validity of our simulation strategy, the structures proposed in reference [4] (conventional normally-on HEMT and normally-off HEMT with fluorine in the $\mathrm{AlGaN}$ layer) were studied using the simulator with the previously tuned parameters. In Figure 4 , the experimental results and the simulated ones are compared and show a clear match.

As mentioned before, the main objective of this work is to propose a new normally-off HEMT structure. At this stage, the above static $I_{\mathrm{d}}\left(V_{\mathrm{gs}}\right)$ simulations used to calibrate the simulator are sufficient.

\section{Results and discussion}

All the simulated structures share the same geometrical and physical properties, as the experimental structure used for calibration (Fig. 1). 


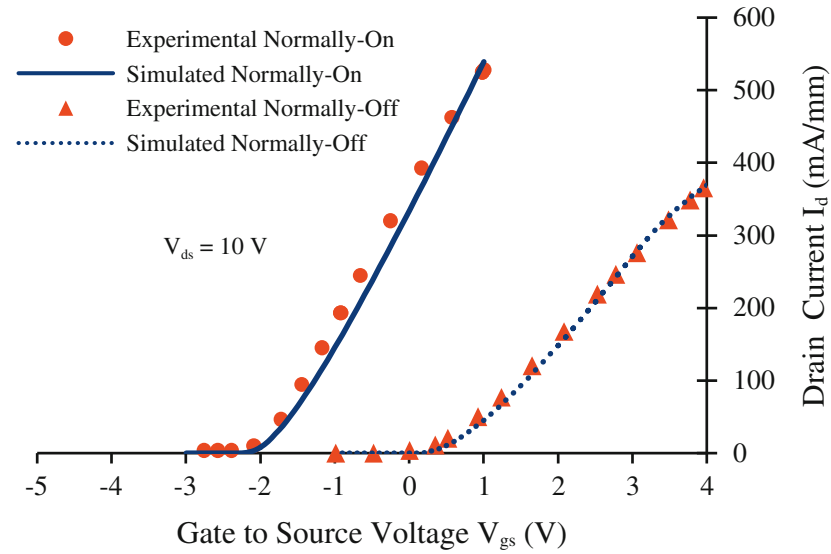

Fig. 4. Comparison between the experimental transfer characteristics $I_{\mathrm{d}}\left(V_{\mathrm{gs}}\right)$ obtained in reference [4] and the simulated ones using the calibrated simulator, for normally-on HEMT and HEMT with fluorine implanted in the barrier layer.

\subsection{Fluorine implantation below the channel}

The effect of fluorine ions, at various concentrations, on the threshold voltage is shown in Figure 5. The distance $d$ between the implanted fluorine ions and the AlGaN/GaN interface is taken equal to $15 \mathrm{~nm}$ (Fig. 3). It is clear that, as the fluorine concentration $\left(F_{\text {imp }}\right)$ increases, the threshold voltage increases. When the fluorine concentration reaches $8 \times 10^{12} \mathrm{~cm}^{-2}$, the HEMT becomes normally-off with a threshold voltage of $0.5 \mathrm{~V}$. To explain this phenomenon, the band diagram, through cut in Figure 3, is shown in Figure 6 . The energy gap between the conduction band and the Fermi level increases as the fluorine concentration increases: this is manifested through the uplifting in the conduction band in the region where fluorine is implanted.

This raise causes the elevation of the conduction band at the $\mathrm{AlGaN} / \mathrm{GaN}$ interface. If the fluorine concentration is strong enough to raise the conduction band at the interface above the Fermi level, normally-off HEMT can be achieved as shown in Figure 5, with a concentration of $8 \times 10^{12} \mathrm{~cm}^{-2}$.

\subsection{Comparison between implantation in the barrier layer and implantation below the channel}

In order to compare the implantation in the barrier layer to that below the channel, the threshold voltage of the conventional normally-on HEMT ("Null") and HEMT with fluorine implanted in AlGaN layer, at various concentrations, is simulated and shown in Figure 5. For a fair comparison, the fluorine ions are located $15 \mathrm{~nm}$ above the $\mathrm{AlGaN} / \mathrm{GaN}$ interface. It can be seen, from Figure 5, that the same threshold voltage of $0.5 \mathrm{~V}$ can be achieved after implantation in both cases. However, the fluorine concentration required to achieve a desired threshold voltage is lower when implantation is performed in the GaN layer/below the channel $\left(8 \times 10^{12} \mathrm{~cm}^{-2}\right)$ rather than in the AlGaN layer/above the channel $\left(14 \times 10^{12} \mathrm{~cm}^{-2}\right)$, making our proposed technique more efficient.

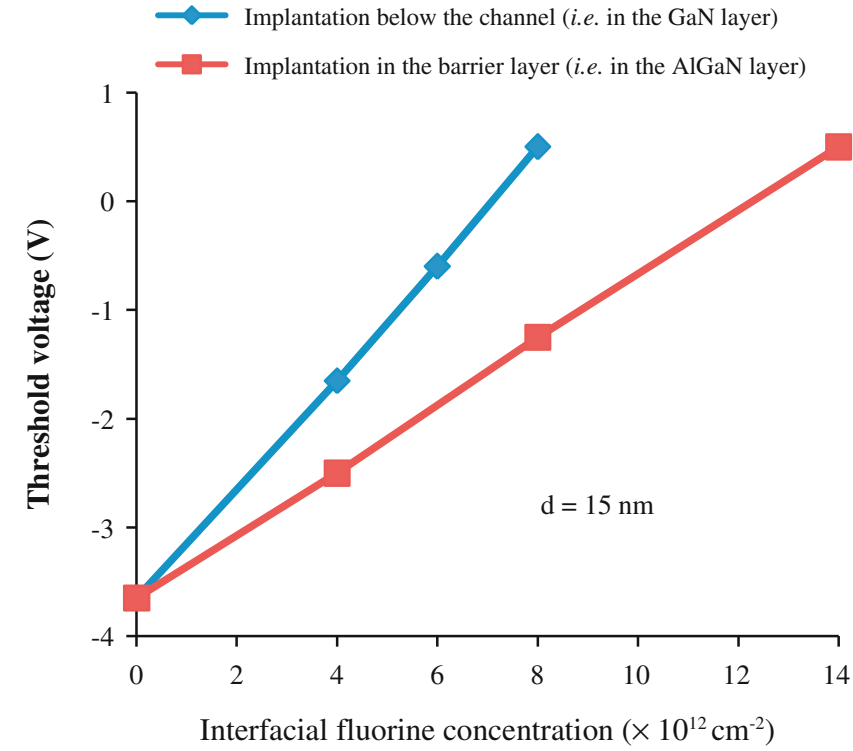

Fig. 5. Simulated $I_{\mathrm{d}}\left(V_{\mathrm{gs}}\right)$ transfer characteristics showing the increase in the threshold voltage with the fluorine concentration.

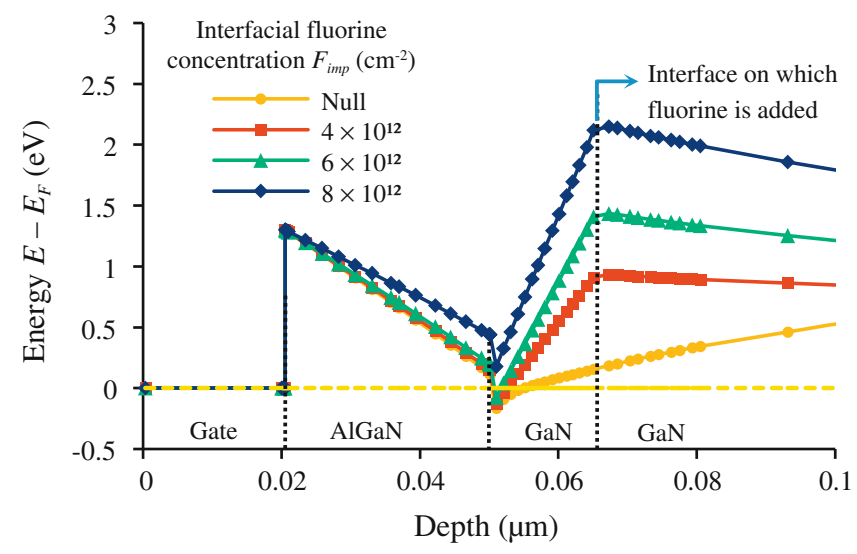

Fig. 6. Conduction band along cut - see Figure 3 - at various fluorine concentrations showing the uplifting of the triangular well at high fluorine concentration.

To explain the better efficiency, the band diagrams for the two structures are compared in Figure 7. In both cases, the threshold voltage is $0.5 \mathrm{~V}$. Since the gate of the HEMT is a Schottky contact, the conduction band at the top of the AlGaN layer is pinned at a fixed energy equal to the Schottky barrier $\Phi_{\mathrm{B}} \cdot \Phi_{\mathrm{B}}=W_{\mathrm{m}}-\chi_{\mathrm{AlGaN}}=1.3 \mathrm{eV}$, where $W_{\mathrm{m}}$ is the work function of the metal and $\chi_{\mathrm{AlGaN}}$ is the electron affinity of the AlGaN layer. This pinning affects the local increase in the potential in the place where fluorine is implanted. However, its influence decreases with increasing the distance between the fluorine and the gate. Since in our proposed technique the distance between fluorine and the gate is higher, the local potential, in the place where fluorine is implanted, is less affected by the boundary conditions. Therefore, relativity smaller concentrations are required to achieve a desired threshold 


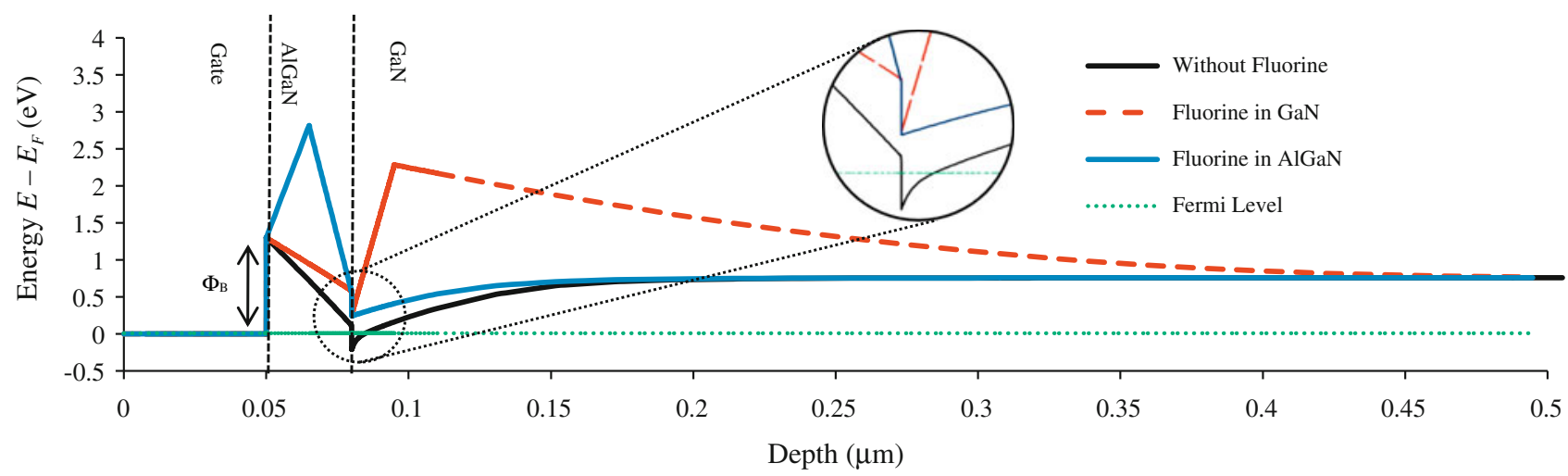

Fig. 7. Band diagrams of HEMT with fluorine implanted in AlGaN at concentration $14 \times 10^{12} \mathrm{~cm}^{-2}$ and HEMT with fluorine in GaN at concentration $8 \times 10^{12} \mathrm{~cm}^{-2}$; both HEMTs exhibit a threshold voltage of $0.5 \mathrm{~V}$.

voltage. Moreover, it can be noted from the band diagram that the confinement of the $2 \mathrm{DEG}$ under the gate is superior in the case of fluorine implanted in GaN (see zoom in Fig. 7).

\subsection{Variations of the threshold voltage with the distance between the fluorine ions and the $A I G a N / G a N$ interface}

Figure 8 shows the variations of the threshold voltage with respect to the distance $d$ between the AlGaN/GaN interface and the fluorine ions, for three different fluorine concentrations. It can be seen that, as the distance increases, the threshold voltage decreases. However, the rate of decrease is more significant after exceeding a certain critical distance $d_{\text {critical }}$. In addition, the results show that $d_{\text {critical }}$ decreases with the increase in the fluorine concentration: for example, the critical distances at fluorine concentration of $6 \times 10^{12}, 7 \times 10^{12}$ and $8 \times 10^{12} \mathrm{~cm}^{-2}$ are 48,33 and $25 \mathrm{~nm}$ respectively. The rate of decrease seems to be independent on the fluorine concentration when $d<d_{\text {critical }}$.

Moreover, the distance $d$ must be below the critical distance for the threshold voltage to increase with increasing the fluorine concentration $\left(F_{\text {imp }}\right)$ (as shown in Fig. 5). For example, $V_{\mathrm{th}}=-0.91 \mathrm{~V}$ for $F_{\mathrm{imp}}=6 \times 10^{12} \mathrm{~cm}^{-2}$ at $d=35 \mathrm{~nm}$. Since at this concentration $d<d_{\text {critical }}$ $(35<48)$, an increase in $F_{\text {imp }}$ from $6 \times 10^{12}$ to $7 \times 10^{12} \mathrm{~cm}^{-2}$ increases $V_{\mathrm{th}}$ from $-0.91 \mathrm{~V}$ to $-0.48 \mathrm{~V}$. However, for $F_{\text {imp }}=7 \times 10^{12} \mathrm{~cm}^{-2}, d>d_{\text {critical }}(35>33)$ : therefore the increase in the fluorine concentration from $7 \times 10^{12}$ to $8 \times 10^{12} \mathrm{~cm}^{-2}$ does not affect the threshold voltage and $V_{\text {th }}$ remains equal to $-0.48 \mathrm{~V}$.

$d_{\text {critical }}$ can be extracted and explained from the band diagram. However, it is worth pointing out three important aspects of the band diagram.

The first is the band bending due to the introduction of charge. Taking the Fermi level at equilibrium as a reference, the conduction band is pushed downwards in the place where positive charge is introduced and upwards in the place where negative charge is introduced.

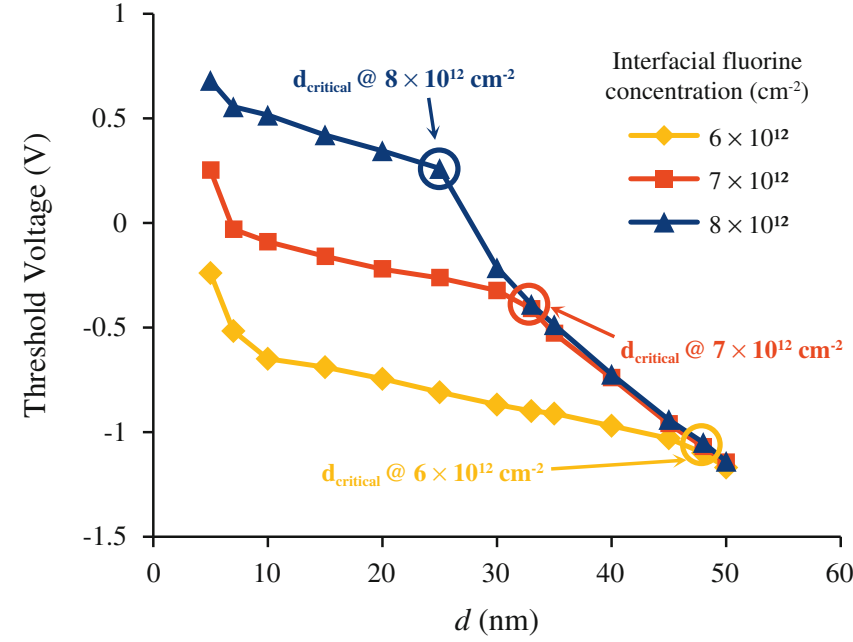

Fig. 8. Variations of the threshold voltage with the distance $d$ between the fluorine ions and the AlGaN/GaN interface at various fluorine concentrations.

The second aspect is the energy gap between the conduction band and the Fermi level $E_{\mathrm{CF}}$. The maximum upward push that can be created by a negative fluorine concentration of $6 \times 10^{12} \mathrm{~cm}^{-2}$ is $E_{\mathrm{CF}, \mathrm{MAX}}=3.404 \mathrm{eV}$. A higher concentration of $8 \times 10^{12} \mathrm{~cm}^{-2}$ can generate higher upward push with $E_{\mathrm{CF}, \mathrm{MAX}}=3.412 \mathrm{eV}$. The difference between $E_{\mathrm{CF}, \mathrm{MAX}}$, at different fluorine concentrations, is very low $(0.008 \mathrm{eV})$ and it is not the reason behind the increase in the threshold voltage with the fluorine concentration. Actually, it is the reason why the threshold voltage does not increase when $d>d_{\text {critical }}$ (will be discussed in more details below).

The third aspect is that $E_{\mathrm{CF}}$, in the region where fluorine is implanted, is strongly affected by the neighboring positive bound charge at the $\mathrm{AlGaN} / \mathrm{GaN}$ interface. While the positive bound charge pulls the conduction band downward, the negative fluorine ions push it upward. $E_{\mathrm{CF}}$ cannot reach $E_{\mathrm{CF}, \mathrm{MAx}}$ unless parting a certain distance from the positive bound charge. Figure 9 shows the band diagram, along cut in Figure 3, with fluorine 


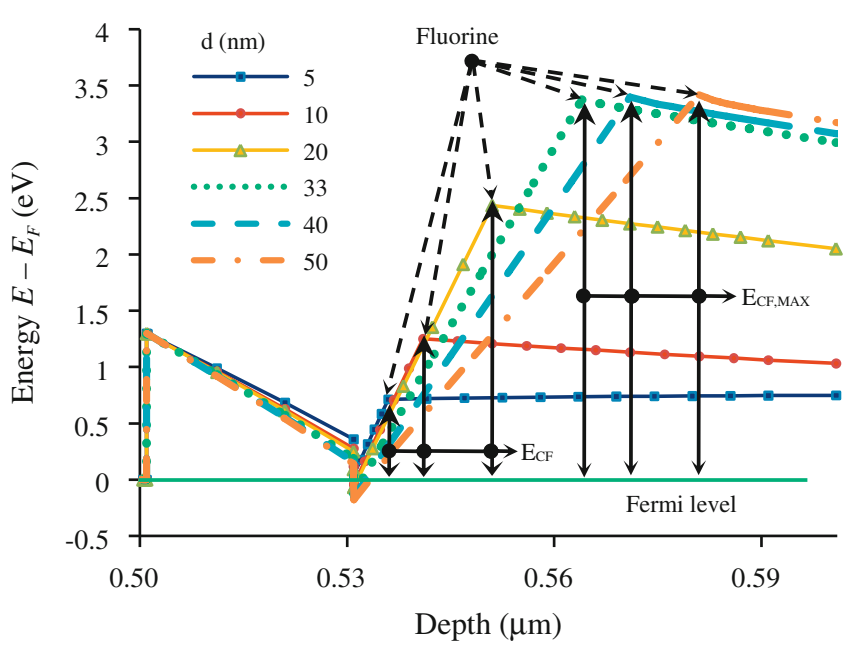

Fig. 9. Band diagram, along cut in Figure 3, with fluorine implanted at various distances from the $\mathrm{AlGaN} / \mathrm{GaN}$ interface. The fluorine concentration is equal to $7 \times 10^{12} \mathrm{~cm}^{-2}$.

implanted at various distances from the AlGaN/GaN interface. The fluorine concentration is taken equal to $7 \times 10^{12} \mathrm{~cm}^{-2}$. The distance at which $E_{\mathrm{CF}}$, in the region where fluorine is implanted, reaches $E_{\mathrm{CF}, \mathrm{MAX}}$, is $d_{\text {critical }}$. $E_{\mathrm{CF}}$ remains constant and equal to $E_{\mathrm{CF}, \mathrm{MAX}}$ for $d>d_{\text {critical }}$. From Figure $9, d_{\text {critical }}$ is equal to $33 \mathrm{~nm}$.

But it is worth noting that, at a given distance below $d_{\text {critical }}$, as the fluorine concentration increases, the upward push in the conduction band increases (see Fig. 6). Therefore, $E_{\mathrm{CF}, \mathrm{MAX}}$ can be reached at relatively short distances. That is why $d_{\text {critical }}$ decreases with increasing the fluorine concentration.

At $d>d_{\text {critical }}$ the threshold voltage does not increase with increasing the fluorine concentration. That is because at that distance, $E_{\mathrm{CF}}$ reaches its maximum value $E_{\mathrm{CF}, \mathrm{MAX}}$, and $E_{\mathrm{CF}, \mathrm{MAX}}$ is barely affected by the increase in the fluorine concentration (recall second aspect: the increase in the fluorine concentration from $6 \times 10^{12} \mathrm{~cm}^{-2}$ to $8 \times 10^{12} \mathrm{~cm}^{-2}$ increases $E_{\mathrm{CF}, \mathrm{MAX}}$ by $\left.0.008 \mathrm{eV}\right)$.

\subsection{Breakdown voltage and off-state current}

In the simulated structures, the density of the bound charge resulting from a $30 \mathrm{~nm}$ AlGaN layer with an $x$-mole fraction of 0.25 is $8.86 \times 10^{12} \mathrm{~cm}^{-2}$. The fluorine equivalent interface charge density required to achieve normallyoff operation, when placed $15 \mathrm{~nm}$ below the channel, is $8 \times 10^{12} \mathrm{~cm}^{-2}$. The existence of high interfacial charge densities in a small spatial region causes convergence problems during the simulation of the breakdown voltage.

To overcome the convergence problem when simulating the off-state current and the breakdown voltage, the density of bound charge was decreased by decreasing the $x$-mole fraction to 0.15 . This decreases the bound charge density to $5.21 \times 10^{12} \mathrm{~cm}^{-2}$.

The decrease in the $x$-mole fraction increases the threshold voltage of the HEMT from $-3.7 \mathrm{~V}$ to $-2 \mathrm{~V}$.

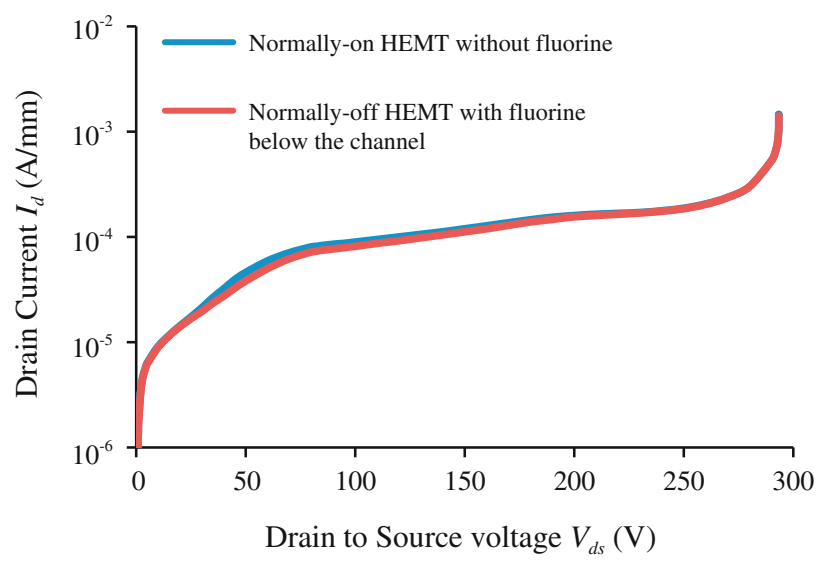

Fig. 10. Simulated $I_{\mathrm{d}}\left(V_{\mathrm{ds}}\right)$ characteristics in the off-state, showing the off-state current and the breakdown voltage of the normally-on HEMT and the normally-off HEMT with fluorine implantation below the channel; both with $x$-mole fraction of 0.15 .

In this case, if fluorine is added $15 \mathrm{~nm}$ below the AlGaN/ GaN interface, a concentration of only $4.2 \times 10^{12} \mathrm{~cm}^{-2}$ is required to achieve normally-off operation with a threshold voltage of $0.5 \mathrm{~V}$.

Figure 10 shows the drain current as a function of the applied drain to source voltage $I_{\mathrm{d}}\left(V_{\mathrm{ds}}\right)$ for the two structures. To study the two HEMTs in the off-state, i.e., below their threshold voltage, the applied gate to source voltage is set to: $V_{\mathrm{gs}}=V_{\mathrm{th}}-1 \mathrm{~V}$. It is clear that neither the vertical breakdown voltage, nor the off-state current are affected by the implanted fluorine ions. A breakdown voltage of $280 \mathrm{~V}$ was obtained in both cases.

\section{Conclusion}

In this work, we have proposed a new normally-off HEMT structure. We suggested the implantation of fluorine ions below the channel region under the $\mathrm{AlGaN} / \mathrm{GaN}$ interface, rather than implanting in the AlGaN layer, above the channel region, as previously proposed in other papers. To analyze the DC performance of this structure, numerical simulation was carried out using ATLAS simulation tool from Silvaco. We have shown that the threshold voltage increases with increasing the fluorine concentration. However, for this increase to happen, the distance between the fluorine ions and the AlGaN/GaN interface $d$ must be below a critical value $d_{\text {critical }}$. $d_{\text {critical }}$ decreases with increasing the fluorine concentration. Moreover, when it comes to the fluorine concentration required to achieve a certain threshold voltage, implantation below the channel is more efficient than the implantation in the barrier layer. Furthermore, with this technique, the confinement of channel electrons below the gate is better and scattering with fluorine ions vanishes, since fluorine ions are buried below the channel layer. Finally, the implantation of fluorine ions below the channel neither affects the vertical breakdown voltage nor the off-state current. 
This work was funded by the Laboratory for Analysis and Architecture of Systems (LAAS-CNRS/France) and by GET/ EDST/UL-AZM \& SAADA association.

\section{References}

1. U.K. Mishra, P. Parikh, Y.-F. Wu, Proc. IEEE 90, 1022 (2002)

2. H. Kambayashi, Y. Satoh, T. Kokawa, N. Ikeda, T. Nomura, S. Kato, Solid-State Electron. 56, 163 (2011)

3. S.D. Burnham, K. Boutros, P. Hashimoto, C. Butler, D.W.S. Wong, M. Hu, M. Micovic, Phys. Status Solidi C 7, $2010(2010)$

4. H. Chen, M. Wang, K.J. Chen, Self-Aligned EnhancementMode AlGaN/GaN HEMTs Using $25 \mathrm{keV}$ Fluorine Ion Implantation, in Device Research Conference (DRC) (IEEE, South Bend, Indiana, USA, 2010), pp. 137-138
5. X. Hu, G. Simin, J. Yang, M.A. Khan, R. Gaska, M.S. Shur, Electron. Lett. 36, 753 (2000)

6. Y. Ohmaki, M. Tanimoto, S. Akamatsu, T. Mukai, Jpn J. Appl. Phys. 45, L1168 (2006)

7. M. Higashiwaki, T. Mimura, T. Matsui, IEEE Trans. Electron Devices 54, 1566 (2007)

8. T. Mizutani, M. Ito, S. Kishimoto, F. Nakamura, IEEE Electron Device Lett. 28, 549 (2007)

9. K.J. Chen, L. Yuan, M.J. Wang, H. Chen, S. Huang, Q. Zhou, C. Zhou, B.K. Li, J.N. Wang, Physics of Fluorine Plasma Ion Implantation For GaN Normally-Off HEMT Technology, in Electron Devices Meeting (IEDM) (IEEE International, Washington, DC, USA, 2011), pp. 19.4.1-19.4.4

10. SILVACO, ATLAS User's Manual Device Simulation Software (Santa Clara, CA, 2011), pp. 330-331

11. Y. Zhang, M. Sun, S.J. Joglekar, T. Fujishima, T. Palacios, Appl. Phys. Lett. 103, 033524 (2013) 